\title{
NMR Study of Mangífera Índica Starch Applied as Food Human
}

\author{
André LBS Bathista* \\ Instituto Federal de Mato Grosso-IFMT, Várzea Grande, Brazil \\ *Corresponding author: André LBS Bathista, Instituto Federal de Mato Grosso-IFMT, Várzea Grande, Brazil. \\ To Cite This Article: André LBS Bathista, NMR Study of Mangífera Índica Starch Applied as Food Human. Am J Biomed Sci \& Res. 2019 - 6(3). AJBSR. \\ MS.ID.001038. DOI: 10.34297/AJBSR.2019.06.001038.
}

Received: 眥 November 2, 2019; Published: 恝 November 25, 2019

\begin{abstract}
Mango seed starch was investigated by 1D and 2D solid state nuclear magnetic resonances (NMR). The basic work focuses the understanding changes in the molecular dynamics of this seed starch, after modification effects on its granule by the addition of acetic acid (AA), ammonium hydroxide $(\mathrm{NH} 4 \mathrm{OH})$ and a mixture of ammonium hydroxide and acetic acid (NH4OH/AA), using the NMR techniques. From the 2D Exchange NMR spectra, recorded at temperature below $\mathrm{Tg}$, in which the molecular motion is restrict for the glucose chains, after the treatments with the three kinds of diluents, the samples showed an amplitude of molecular motion, even below $\mathrm{Tg}$, which was not observed for the sample in nature, due to the changes in the molecular mobility in the starch granule after the treatments, showing that the modified starches present new molecular mobility, because new interactions and chains ordination has occurred after these processes, these changes were also confirmed by VCT and PUREX results.
\end{abstract}

Keywords: NMR; Starch; Mango; Human

\section{Introduction}

The quality of starches and their derivatives is an important factor for their use in food industry. Considering that starches are the major chemical compounds of food such as cereals, they are a subject of many studies. Their characterization has a great interest in the entire world [1]. It is known that each starch presents its own properties, such as composition, crystal type, granule organization and glass temperature, as well as gelatinization process. Thus, the nature of starches implies their characteristics. Studies on starch focus the identification of crystal type and composition. Other studies involve the understanding of chemical structure and molecular dynamics. Analytical techniques, including nuclear magnetic resonance (NMR) via nuclear relaxation time has been well stablished to be used as a methodology to evaluate heterogeneous samples like starch. In this work basic NMR techniques, such as Magic Angle Spinning (MAS); Cross-Polarization (CP); Cross-Polarization Magic Angle Spinning (CPMAS) and Variable Contact Time (VCT) [2-19], together with two-dimensional Exchange (2D exchange) and Pure-Exchange (PUREX) have been used. Those techniques are very helpful to understand the chemical structural differences and permit to evaluate the molecular dynamic of starch compounds in different time scales. The MAS was used with short delay between 90 o pulses, to investigate the high mobility domain. The CPMAS was applied to have a response of entire sample. The VCT was employed to evaluate the efficiency of polarization transfer and consequently to identify the mobile domains. The 2D Exchange was carried out to evaluate the amplitude of the molecular mobility of the entire starch molecule. The 1D PUREX pulse sequence was recorded to detect investigate the mobile fraction of the starch samples after they have been treated with acetic acid (AA) and $\mathrm{NH}_{4} \mathrm{OH}$. The focus of this work is on understanding the changes in the molecular dynamics of mango seed starch, after modification effects on its granule by the addition of acetic acid (AA), ammonium hydroxide $\left(\mathrm{NH}_{4} \mathrm{OH}\right)$ and a mixture of ammonium hydroxide and acetic acid $\left(\mathrm{NH}_{4} \mathrm{OH} / \mathrm{AA}\right)$, using the NMR techniques mentioned before.

\section{Experimental}

The starch was modified using two diluents: $\mathrm{NH}_{4} \mathrm{OH} 0.1 \mathrm{M}$ and acetic acid (AA) $0.1 \mathrm{M}$. The three/four samples were named as mango starch (in nature) (1); mango starch/ AA (2), mango starch/ $\mathrm{NH}_{4} \mathrm{OH}(3)$ and mango starch/AA (4). 


\section{NMR Background}

This section gives a brief introduction about the exchange methods employed in this work. Detailed descriptions of the methods described below are found in references [20-25]. 2D Exchange NMR. Figure 1a shows the pulse sequence for the static 2D exchange experiment. In this experiment the orientation dependent NMR frequencies are monitored before (0) and after () a mixing time 0 when molecular reorientations can occur. If no exchange happens, the resulting 2D spectrum is purely diagonal. In contrast, if slow molecular reorientations occur during, off diagonal intensities are observed. The shape of the 2D spectrum strongly depends on motional amplitude, making possible to distinguish whether the motion involves small or large amplitude reorientations. The identification of the motional amplitude can be made directly from the 2D spectrum without the need of any specific model.

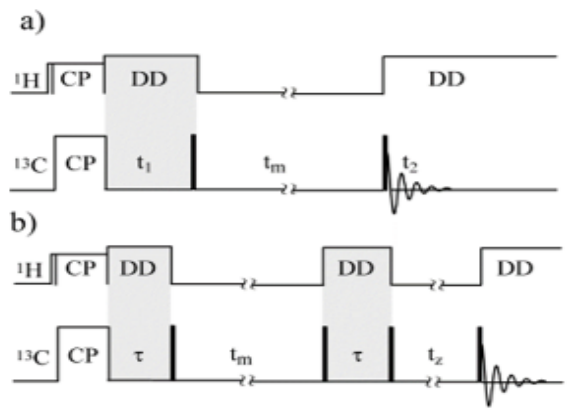

Figure 1: a) Pulse sequence used in the exchange experiments, $\left(90^{\circ}\right.$ pulses are filled white)

b) Pulse sequence used in the PUREX experiments, $\left(90^{\circ}\right.$ pulses are filled white).

1D Pure Exchange (PUREX) method. The pulse sequence for the 1D PUREX NMR experiment is shown in Figure 1b. 1D PUREX is a stimulated-echo experiment that detects only segments reorienting in the exchange frequency window ( $\mathrm{Hz}$ to $\mathrm{kHz}$ ). This is achieved by monitoring the signal reduction due to changes in the orientation-dependent chemical-shift frequencies, which results from segmental reorientations that take place during a long $(\sim \mathrm{ms}$ to $\mathrm{s}$ ) mixing time (tm). The tm dependence of the ratio between each NMR line intensity, $\mathrm{S}(\mathrm{tm}, \tau)$, and the respective line intensity obtained from a control experiment, S0 $(0, \tau)$, (that do not encode any molecular motion) provides the two-time correlation function of the slow molecular motions [21].

\section{NMR Experiments}

The 13C NMR solid state spectra were obtained on a VARIAN INOVA 400 spectrometer, operating at $100 \mathrm{MHz}$ for carbon-13. The MAS conditions was spectral width: $30.000 \mathrm{~Hz}$; acquisition time: $0.04 \mathrm{~s}$; pulse width $90^{\circ}$, recycle delay $0.3 \mathrm{~s}$ and number of transients 5000. For CPMAS the spectral width: $30.000 \mathrm{~Hz}$; acquisition time: $0.04 \mathrm{~s}$; pulse width $90^{\circ}$, recycle delay $3 \mathrm{~s}$ and number of transients 512. The VCT conditions used were the same for the ${ }^{13} \mathrm{C}$. A 7-mm static double resonance probe head was used $\pi / 2$ pulse lengths of $3.8 \mathrm{~ms}$ and $4.0 \mathrm{~ms}$ was applied for ${ }^{13} \mathrm{C}$ and ${ }^{1} \mathrm{H}$, respectively. The proton decoupling field strength was approximately $60 \mathrm{kHz}$, crosspolarization time of 1.0ms. In 2D Exchange experiments 64 to 128 increments in were acquired using a total of 32 scans per point. The mixing times for 2D experiments were $200 \mathrm{~ms}$. The 1D Pure exchange NMR (1D PUREX) experiments were performed also using mixing times of $200 \mathrm{~ms}$ and $250 \mathrm{~ms}$. CPMAS with a range of contact time established as 200 to $8000(\mathrm{~ms})$. The processing used was zero filling and line broadening was 50 .

\section{Results and Discussion}

\section{NMR CPMAS}

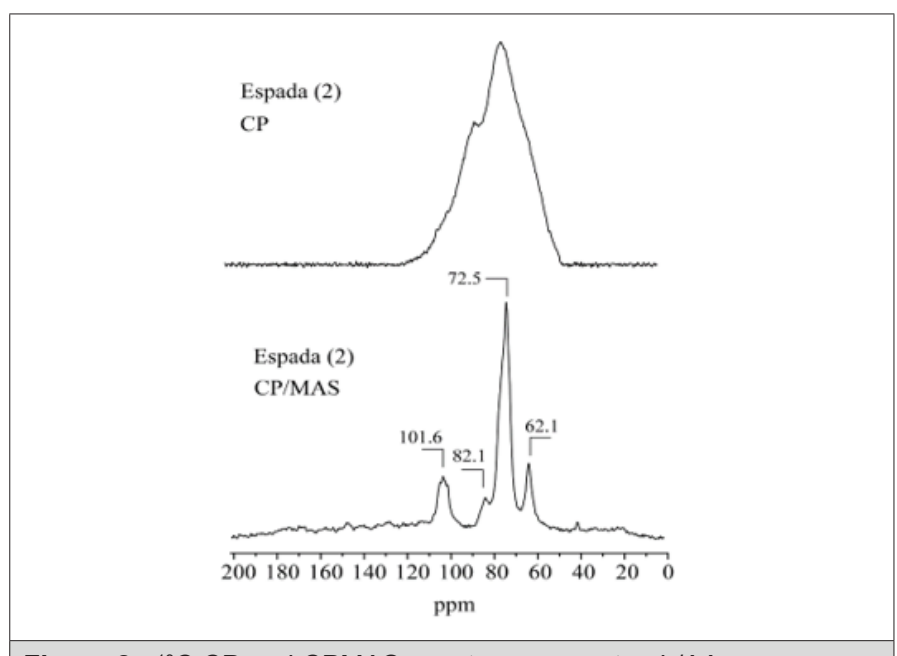

Figure 2: ${ }^{13} \mathrm{C} \mathrm{CP}$ and CPMAS spectra mango starch/AA.

A comparison of the ${ }^{13} \mathrm{C}$ CPMAS spectra with and without rotation for all samples showed the distribution shape of the resolved carbon-13 signals. The 13C CPMAS of mango starch presents signals located at: 62ppm $\left(\mathrm{CH}_{2}-\mathrm{OH}\right), 71.3 \mathrm{ppm}(\mathrm{CH}-\mathrm{OH})$ and 101ppm (C-O-C). Figures 2-4 shows a comparison between ${ }^{13} \mathrm{C}$ CPMAS spectra of mango starch/AA, mango starch/ $\mathrm{NH}_{4} \mathrm{OH}$ and starch/ $\mathrm{NH}_{4} \mathrm{OH} / \mathrm{AA}$ respectively. Some differences between the two spectra were identified, such as: 1-the signal shapes are different, especially for the anomeric carbon; 2-the chemical shift value of $\mathrm{C}-\mathrm{O}-\mathrm{C}$ changed after both types of starch treatment, the chemical shift value detected for the starch treated with $\mathrm{NH}_{4} \mathrm{OH}$ is higher than the value found for the starch treated by acetic acid, this change can be due to the change in the starch sample morphology, which influences the chains arrangements and packing. And finally, three small signals were detected between 20-40ppm when the starch was treated with $\mathrm{NH}_{4} \mathrm{OH}$, which can be derived from others minor components like glutens, for example. Figures 2-5 shows the ${ }^{13} \mathrm{C} \mathrm{CP}$ spectra of starch recorded as a function of treatments. The line width in the spectrum obtained after the treatment is slightly higher than in the MAS spectrum (Figure 6). Pronounced line shape changes are observed with respect to the static CP spectrum of treated starch (Figure 3). This is an indicative of large amplitude motions with rates exceeding the width of $\mathrm{CP}$ 
spectrum, i.e. above $3 \mathrm{kHz}$ (Table 1). The overlapping signal around 68-78ppm is associated with $\mathrm{C}_{2}, \mathrm{C}_{3}, \mathrm{C}_{5}$. The $\mathrm{C}_{1}$ resonances of both in nature and modified mango starches are triplets for espada (1) and (2), which is a typical A-type characteristic [2] (Figure 6)

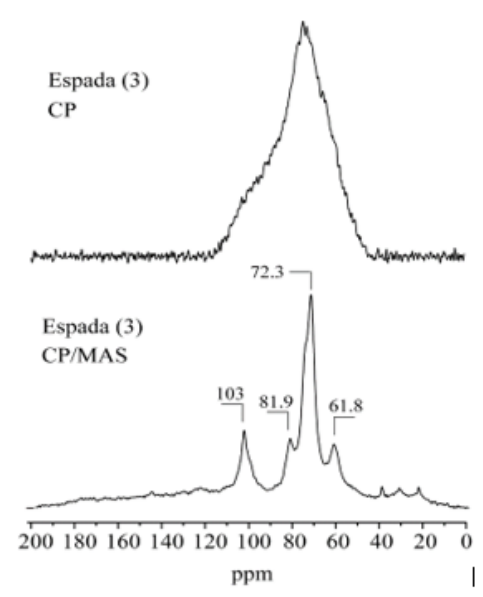

Figure 3: ${ }^{13} \mathrm{C}$ CPMAS spectra mango starch/ $\mathrm{NH} 4 \mathrm{OH}$.

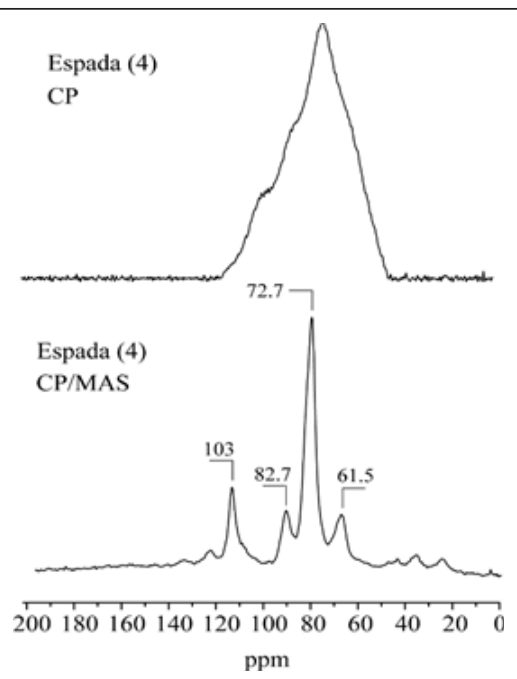

Figure 4: ${ }^{13} \mathrm{C}$ CPMAS spectra mango starch/NH4OH/AA.

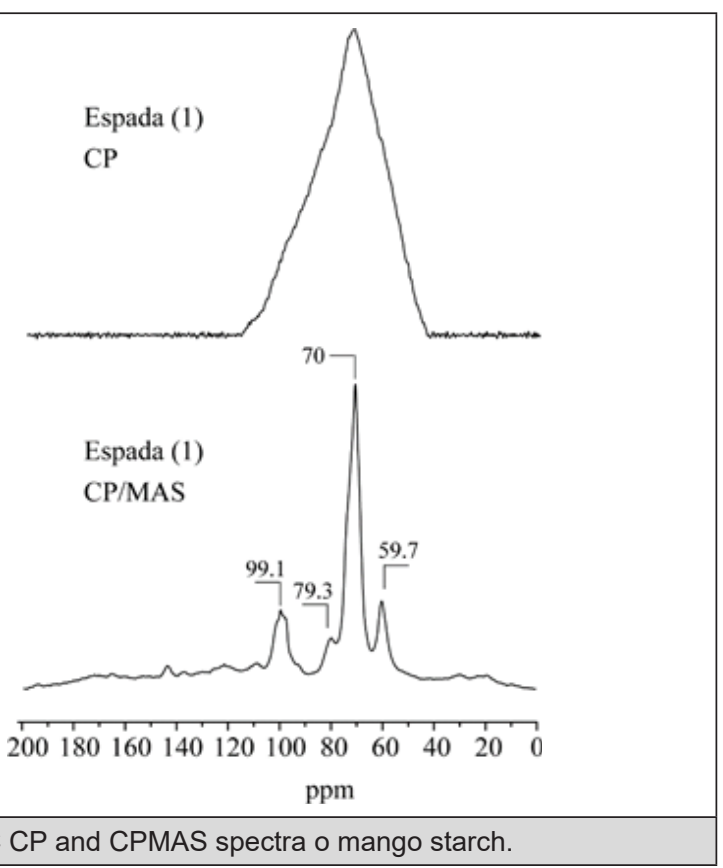

Figure $5:{ }^{13} \mathrm{C}$ CP and CPMAS spectra o mango starch.
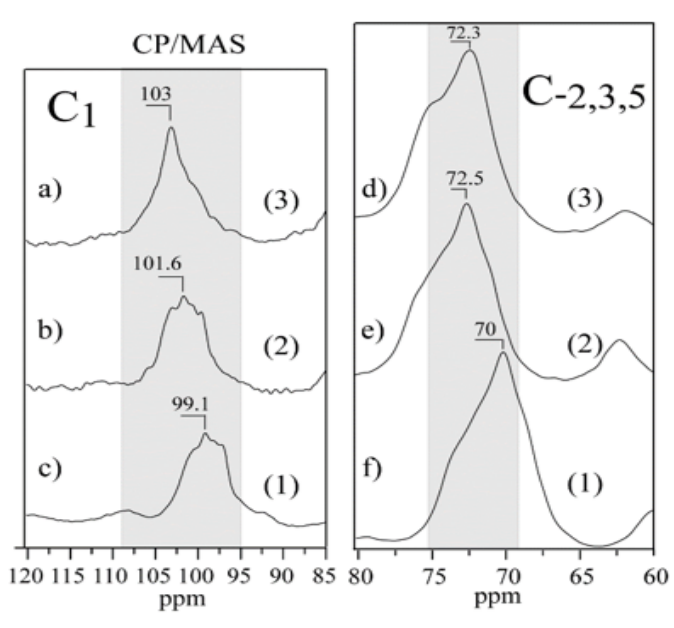

Figure 6: ${ }^{13} \mathrm{C}$ CPMAS comparison of $\mathrm{C} 1$ glucose a) starch/ $\mathrm{NH} 4 \mathrm{OH}$ b) starch/AA and c) native starch.

\begin{tabular}{|c|c|c|c|c|}
\hline Table 1: 13C CP/MAS NMR chemical shifts of nature and modified mango starches. & \multicolumn{1}{l|}{ C2,3,5 } & C6 \\
\hline Mango & C1 & C4 & (ppm) & 59.7 \\
\hline-1 & Chemical & Shifts & 70 & 62.1 \\
\hline-2 & 99.1 & 79.3 & 72.5 & 61.8 \\
\hline-3 & 101.6 & 82.1 & 72.3 & 61.5 \\
\hline-4 & 103 & 81.9 & 72.7 & \\
\hline
\end{tabular}

\section{NMR 2D Exchange}

In order to check whether molecular motion on longer time scale, 2D exchange NMR techniques were applied. Figures 5 shows the 2D Exchange spectra acquired with mixing times of $200 \mathrm{~ms}$ for the sample (3) and (4) at $25^{\circ} \mathrm{C}$ (below $\mathrm{Tg}$ ). The temperatures were chosen to cover the range where the motion that occurs in the slowmotion regime [6]. The $2 \mathrm{D}$ spectrum obtained at $25^{\circ} \mathrm{C}$ clearly shows two distinct regions, being the spectral region attributed to the ${ }^{13} \mathrm{C}$ nuclei in granule starch (1) fully diagonal while the signal of the $\mathrm{C}_{1-6}$ groups (3) is typical for segments executing isotropic motion. This is basically the same behavior observed for (4). This confirms that the hindrance to the molecular motion of the glucose groups close to the granule structure might be effective even for higher temperatures. This different behavior can be understanding if the distribution of correlation times of the motions involved in glass 
transition of starch is smaller for treated samples as compared to non-treated. Apparently, due to the dense packing of chains in native Espada starch, molecular motions are intrinsically absent (Figure 7). The exchange pattern (off-diagonal intensity) indicates the existence of molecular motions on a time scale of $200 \mathrm{~ms}$ (compared Figure 8 Espada). Note that for all carbons $\left(\mathrm{C}_{1}-\mathrm{C}_{6}\right)$ the exchange intensity for sample (3) and (4) show the reorientation during $\mathrm{tm}$, and for diagonal intensity indicate motion above the time scale.

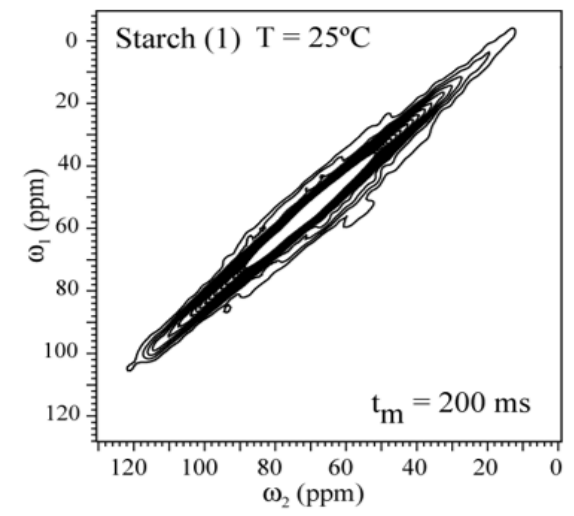

Figure 7: Results of 2D exchange experiments performed for Espada Starch (1) the slowing down of the molecular motion is observed by the smaller number of contour lines in (1). The contour levels were taken from 5 to $20 \%$ of the maximum height.

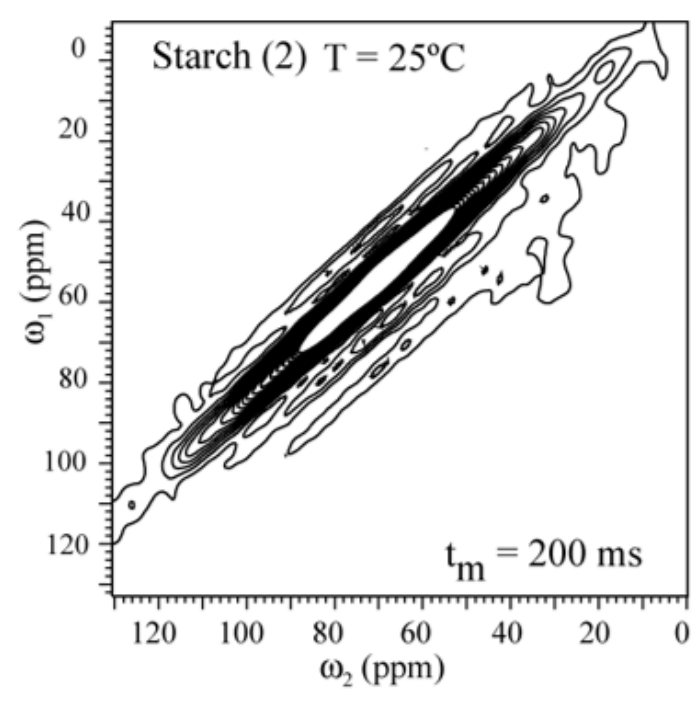

Figure 8: Results of 2D exchange experiments performed for Espada Starch (2).

Table 2: $\mathrm{T} 1_{\rho}(\mathrm{ms})$ of nature and modified mango starches.

\begin{tabular}{|c|c|c|c|}
\hline Mango & C1 & C2,3,5 & C6 \\
\hline & time & (ms) & \\
\hline-1 & 3.16 & 4 & 3.33 \\
\hline-3 & 4.29 & 3.96 & 2.37 \\
\hline
\end{tabular}
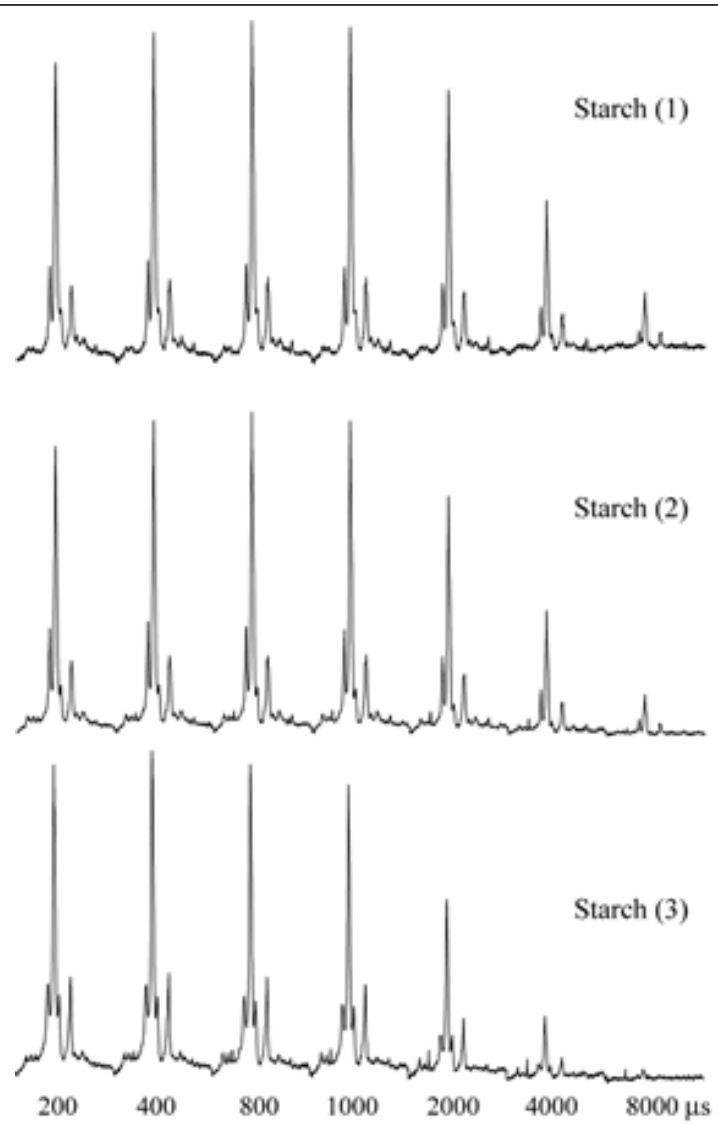

Figure 9: 1D VCT performed for Espada Starch a) (1), b) (2), c) (3). 
The 2D Exchange spectra of the (3) and (4) samples show a higher amplitude of molecular motion comparing to the (1) and (2) samples. These results can be attributed to the type of granule breaking, which influences direct in the molecular motion. For the samples (3) and (4) the molecular motion in the time scale of milliseconds to seconds is observed in out of the diagonal in the spectra. By contrary for the samples (1) e (2) the molecular motion in the same time scale is not detected out of the diagonal. The motions detected in the range of microseconds to milliseconds (ms-ms) can be done by VCT experiment, which detect the different types of glucose mobility in the range of $\mathrm{kHz}$, according to the intensity of the spectral array (Figure 9). From VCT experiment both treatments make the mango starch more rigid in this time scale when is compared to sample in nature, the treatment with AA became the starch more rigid than the treatment with $\mathrm{NH}_{4} \mathrm{OH}$ (Table 2).

The PUREX 1D technique could be used the obtaining of the molecular motion correlations in a scale range between $1 \mathrm{~ms}$ to $1 \mathrm{~s}$. The information's on motion time scale (ms-s) can be obtained from the peak intensities of the 1D PUREX spectra as a function of mixing time, tm. The 1D PUREX intensity of each point of Figure 10 was integrated from 120 to $20 \mathrm{ppm}$ and it was also normalized according to the equation: $E\left(t_{m}, \tau\right)=\left(S-S_{0}\right) / S_{0}$ The integration was realized in order to only detect the molecular motion amplitude, including all carbons of glucose $\left(\mathrm{C}_{1-6}\right)$ chains. Thus, the plot obtained from the ratio $\left(S-S_{0}\right) / S_{0}$ as a function of tm can be used to characterize the dynamic motion, therefore without relaxation effect due to the normalization. The mixing time employed in this experiment was $t_{m}>\tau_{c}$ in order to detect the slow molecular motion, which may occur in the range of the PUREX observation. From the 1D PUREX curves, the mixing time $t m=200 \mathrm{~ms}$ was the ideal time for the measurement of the 2D Exchange according to the 1D PUREX curve slope (Figure 11,12).

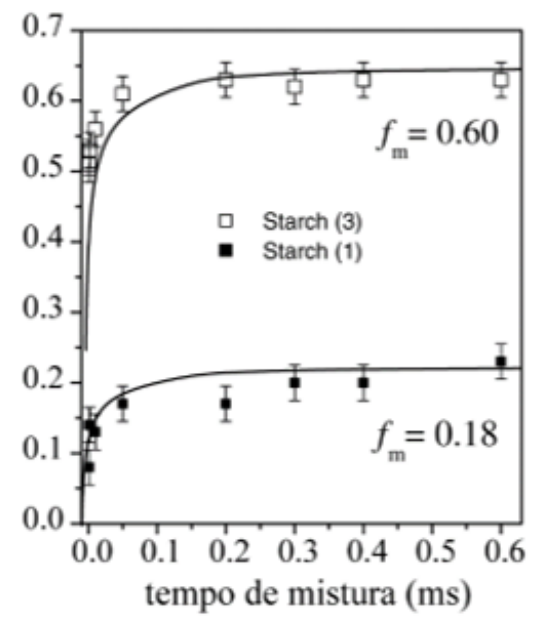

Figure 10: 1D Pure-exchange experiments performed for Espada Starch a) (1) and (3).
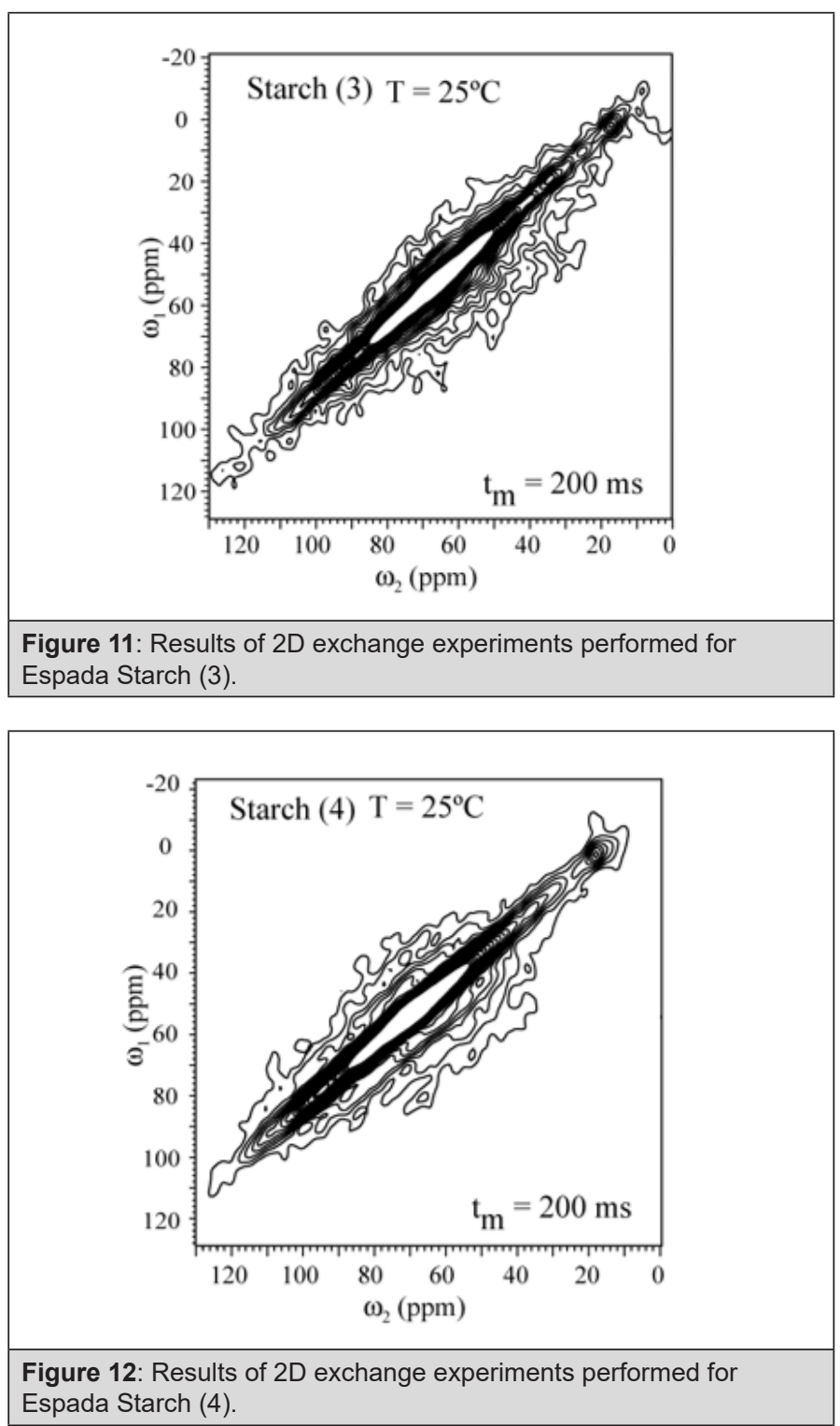

\section{Conclusion}

According to the main purpose of this work, there was detected an opening of the starch granule as a function of the different diluents. The 2D Exchange NMR spectra were proposal recorded at temperature below $\mathrm{T}_{\mathrm{g}}$, in which the molecular motion of these samples is restricted because these chains are in a rigid lattice. After the treatments with the three diluents, the samples showed an amplitude of molecular motion, even below $\mathrm{T}_{\mathrm{g}}$, which was not observed for the sample (1), showing that the modified starches present new molecular mobility due to the new interactions and chains ordination after the treatments, which was confirmed by VCT, 2D Exchange and PUREX results.

\section{References}

1. Scott G (2000) 'Green' Polymers. Polymer Degradation and Stability 68: 1-7.

2. Atichokudomchai N, S Varavinit, P Chinachoti (2004) A study of ordered structure in acid-modified tapioca starch by 13C CP/MAS solid-state NMR. Carbohydrate Polymers 58(4): 383-389. 
3. Cheetham NWH, LP Tao (1998) 36(4): 285-292.

4. Costa PM (2003) IX Encontro de Usuários de Ressonância Magnética Nuclear. Angra dos Reis, Auremn, Brazil.

5. Kulik AS, JRC Decosta, J Haverkamp (1994) Water organization and molecular mobility in maize starch investigated by two-dimensional solid-state NMR. Journal of Agricultural and Food Chemistry 42(12): 2803-2807.

6. Kulik AS, J Haverkamp (1997) Molecular mobility of polysaccharide chains in starch investigated by two-dimensional solid-state NMR spectroscopy. Carbohydrate Polymers 34(1-2): 49-54.

7. Laignel B, Christophe Bliard, Georges Massiot, Jean Marc Nuzillard (1997) Proton NMR spectroscopy assignment of d-glucose residues in highly acetylated starch. Carbohydrate Research 298(4): 251-260.

8. Kulik AS, J Haverkamp (1997) Molecular mobility of polysaccharide chains in starch investigated by two-dimensional solid-state NMR spectroscopy. Carbohydrate Polymers 34(1-2): 49-54.

9. Le Botlan D, Yannick Rugraff, Cyril Martin, Paul Colonna (1998) Quantitative determination of bound water in wheat starch by time domain NMR spectroscopy. Carbohydrate Research 308(1-2): 29-36.

10. LI S, LC Dickinson, P Chinachoti (1996) Proton Relaxation of Starch and Gluten by Solid-State Nuclear Magnetic Resonance Spectroscopy. Cereal Chemistry 73(6): 736-743.

11. Mendes da Silva CE, CF Ciacco, GE Barberis, WMR Solano, C Rettori (1996) Starch Gelatinization Measured by Pulsed Nuclear Magnetic Resonance. Cereal Chemistry 73(3): 297-301.

12. Morgan KR, RH Furneaux, NG Larsen (1995) Solid-state NMR studies on the structure of starch granules. Carbohydrate Research 276(2): $387-$ 399.

13. Tang HR, A Brun, B Hills (2001) A proton NMR relaxation study of the gelatinisation and acid hydrolysis of native potato starch. Carbohydrate Polymers 46(1): 7-18.
14. Tang HR, J Godward, B Hills (2000) The distribution of water in native starch granules-a multinuclear NMR study. Carbohydrate Polymers 43(4): 375-387.

15. Tavares MIB (2004) Journal Applied Polymer Science 93(4): 2151-2154.

16. Tavares MIB (2002) $6^{\text {th }}$ International Conference on Applications of Magnetic Resonance in Food Science. Paris-France.

17. Tavares MIB, André Luis BS Bathista, Emerson O Silva, Nicolau P Filho, José S Nogueira (2003) A molecular dynamic study of the starch obtained from the Mangifera indica Cv. Bourbon and Espada seeds by $13 \mathrm{C}$ solid state NMR. Carbohydrate Polymers 53(2): 213-216.

18. Tavares MIB (2001) The $2^{\text {nd }}$ Alpine Conference on Solid-State NMR. Chamonix-Mont Blanc-France: International Society of magnetic Resonance and the Groupement Ampere.

19. Tavares MIB (2002) VII Jornada Brasileira de Ressonância Magnética. Auremn, Maringá Brazil.

20. De Azevedo ER, Tito José Bonagamba (2001) Molecular dynamics and local molecular conformation in solid materials studied by nuclear magnetic resonance. Instituto de Física de São Carlos, Universidade de São Paulo, São Carlos, Brazil pp. 197.

21. De Azevedo ER, TJ Bonagamba, K Schmidt Rohr (2000) Journal of Magnetic Resonance 142: 86-89.

22. De Azevedo ER (2003) Chem Mater 15(10): 2070-2078.

23. Kaufmann S (1990) Journal of Chemical Physics 93(1): 197-214.

24. Schmidt Rohr K, HW Spiess (1994) Multidimensional Solid-State NMR and Polymers. Academic Press, San Diego CA, USA pp. 478.

25. Wefing S, S Kaufmann, HW Spiess (1988) Two-dimensional exchange NMR of powder samples. II. The dynamic evolution of two-time distribution functions. Journal of Chemical Physics 89(3): 1234-1244. 